\title{
Limit on Non-Standard Interactions from the atmospheric neutrino data
}

\author{
Gaku Mitsuka* (The Super-Kamiokande collaboration) \\ Institute for Cosmic Ray Research, University of Tokyo \\ 5-1-5 Kashiwa-no-Ha, Kashiwa City \\ Chiba, 277-8582, Japan \\ E-mail: mituka@icrr.u-tokyo.ac.jp
}

\begin{abstract}
We searched for non-standard neutrino interaction with the whole atmospheric neutrino data in Super-Kamiokande I and II. Neutrino oscillations in atmospheric neutrinos as well as that in the solar and reacter neutrinos play a very important role in particle physics because they present the evedence for physics beyond the standard model. Focussing on the existing atmospheric neutrino data, they are explained very well by $v_{\mu} \rightarrow v_{\tau}$ oscillation scheme, here we can use the robustness of the imprementation in order to obtain tight limits on a lot of mechanisms proposed alternative to the neutrino oscillations. Our main interest in this paper is on the placement of stringent limits on one possible alternative solution, non-standard neutrino interaction, in $v_{\mu} \rightarrow v_{\tau}$ sector with 2flavor atmospheric neutrino framework.
\end{abstract}

10th International Workshop on Neutrino Factories, Super beams and Beta beams June 30 - July 52008

Valencia, Spain

\footnotetext{
* Speaker.
} 


\section{Introduction}

A lot of attemps have been proposed to explain the atmospheric neutrino problem without neutrino oscillation hypothesis[i]. One possible solution to the problem is non-standard neutrino interacton(NSI) where the neutrinos possess non-standard interactions with matter in the Earth, which shows a good agreement with the Fully and Partially contained events. NSI we focus here are composed of flavor-changing(FC) and non-universal(NU) processes arised naturally in the presence of heavy mediator fields[2].

Our main interest in this paper is on the placement of stringent limits on NSI in $v_{\mu} \rightarrow v_{\tau}$ sector with 2 flavor atmospheric neutrino framework. In the forthcoming accelerator experiments, for exapmle the T2K experiment, one of the main goal is the precision measurement of the $v_{\mu} \rightarrow v_{\tau}$ neutrino oscillation parameters. However, the present loose limits on NSI will be the interference of this purpose. Therefore, this work is very important so as to obtain the higher sensitivity in next-generation neutrino oscillation experiments.

We will first introduce the formalism in Sec.2. In Sec.3, we will summarize the atmospheric neutrino data and the Monte Carlo simulations. In Sec.4, we show the analysis with the combined probability where neutrino oscillations coexist with NSI with matter, and derive limits on NSI parameters. We conclude this work in Sec.5.

\section{Formalism}

We show the formalism where neutrino oscillations coexist with NSI, we call it as Hybrid model. In this work, we follow the formalism by M.C. Gonzalez-Garcia and M. Maltoni[ß], where the propagation of neutrinos(+) and antineutrinos(-) is governed by the following Hamiltonian:

$$
H \equiv \frac{\Delta m^{2}}{4 E} U_{\theta}\left(\begin{array}{cc}
-1 & 0 \\
0 & 1
\end{array}\right) U_{\theta}^{\dagger} \pm \sqrt{2} G_{F} N_{f}(\vec{r}) U_{\xi, \pm \eta}\left(\begin{array}{cc}
-1 & 0 \\
0 & 1
\end{array}\right) U_{\xi, \pm \eta}^{\dagger}
$$

where $N_{f}(\vec{r})$ is the number density of the fermion $f$ along the path $r$ of the neutrinos propagating in the Earth. The matrics $U_{\theta}$ and $U_{\xi, \pm \eta}$ are given by:

$$
\begin{aligned}
U_{\theta} & =\left(\begin{array}{cc}
\cos \theta & \sin \theta \\
-\sin \theta & \cos \theta
\end{array}\right) \\
U_{\xi, \pm \eta} & =\left(\begin{array}{cc}
\cos \xi & \sin \xi e^{ \pm i \eta} \\
-\sin \xi e^{\mp i \eta} & \cos \xi
\end{array}\right) \\
\xi & =\frac{1}{2} \arctan \left(\frac{\varepsilon}{\varepsilon^{\prime} / 2}\right)
\end{aligned}
$$

where possible non-vanishing relative phase $\eta$ is considered. $\sqrt{2} G_{F} N_{f}(\vec{r}) \varepsilon$ is the amplitude of the flavor-changing neutral current(FCNC) process $v_{\mu}+f \rightarrow v_{\tau}+f$, while $\sqrt{2} G_{F} N_{f}(\vec{r}) \varepsilon^{\prime}$ is the amplitude of lepton non-universality(NU).

If the matter profile in the Earth is constant along the neutrino trajectory, $P_{v_{\mu} \rightarrow v_{\mu}}$ is expressed as:

$$
P_{v_{\mu} \rightarrow v_{\mu}}=1-P_{v_{\mu} \rightarrow v_{\tau}}=1-\sin ^{2} 2 \Theta \sin ^{2}\left(\frac{\Delta m^{2} L}{4 E} R\right)
$$


where the effective mixing angle $\Theta$ and NSI's correction factor to oscillation wavelength, $R$, are given as

$$
\begin{aligned}
\sin ^{2} 2 \Theta & =\frac{1}{R^{2}}\left(\sin ^{2} 2 \theta+R_{0}^{2} \sin ^{2} 2 \xi+2 R_{0} \sin 2 \theta \sin 2 \xi \cos \eta\right) \\
R & =\sqrt{1+R_{0}^{2}+2 R_{0}(\cos 2 \theta \cos 2 \xi+\sin 2 \theta \sin 2 \xi \cos \eta)} .
\end{aligned}
$$

$R_{0}$ gives the ratio between standard oscillation and NSI to the oscillation wavelength

$$
R_{0}= \pm \frac{\Delta \delta}{2} \frac{4 E}{\Delta m^{2}}
$$

NSI effect $\Delta \delta$ is given as:

$$
\begin{aligned}
\Delta \delta & =2 \sqrt{2} G_{F} N_{f}(\vec{r}) F \\
& \equiv 4.58 \times 10^{-22}\left(2-Y_{p}\right) \frac{\rho(\vec{r})_{E a t r h}}{3 g / \mathrm{cm}^{3}} F \mathrm{GeV} \\
F & =\sqrt{|\varepsilon|^{2}+\frac{\varepsilon^{\prime 2}}{4}}
\end{aligned}
$$

We use PREM model for the matter density profile and a chemical composition with protonnucleon ratio $Y_{p}=0.497$ and 0.468 in the mantle and core, respectively. Fermion $f$ in the above fornulas is assumed to be down-quark.

\section{Atmospheric neutrino data and Monte Carlo simulation}

\subsection{Atmospheric data sample}

In this analysis, Fully contained(FC), Partially contained(PC), and Upward-going $\mu$ (UPMU) data during Super-Kamiokande(SK) I period(1489.2 days exposure for FC and PC, 1645.9days for UPMU) and SK-II period(798.6days for FC and PC, 827.7days for UPMU) are used. Since the resonctruction of long path length muon is less sensitive to the detector condition, livetime of UPMU data is larger than tha of FC and PC data.

\subsection{Monte Carlo simulation}

Expectation of atmospheric neutrino events in SK is calculated by a Monte Carlo simulation, which consists of three components: atmospheric neutrino flux, neutrino interaction, detector simulation.

In our neutrino flux simulation, the HKKM06 flux[ [4] is adopted, although the HKKM03 flux was used in the past oscillation analysis with SK-I.

The neutrino inteaction with nucleon or nucleus in water are simulated using NEUT program library[[]. NEUT was first developed in the Kamiokande experiment and has been updated continously. Recently a lot of improvements are adopted to take account of the progress in the understanding of neutrino interactions in the last decade. 


\section{Analysis}

We present the results of the standard oscillation+NSI hybrid model. As discussed in the section 2 we have five free parameters: $\Delta m^{2}, \theta, \Delta \delta, \varepsilon^{\prime},|\varepsilon|$ and $\arg (\varepsilon)$.

Fig. 1 shows the allowed region of standard oscillation parameters: $\Delta m_{23}^{2}$ and $\sin ^{2} 2 \theta_{23}$.

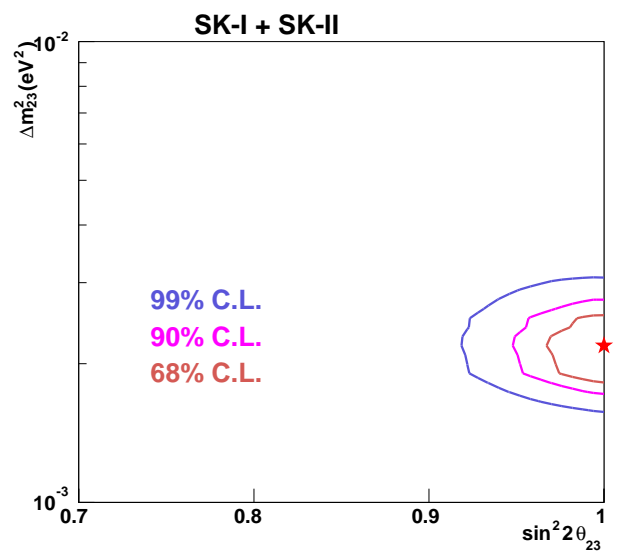

Figure 1: Allowed region of standard oscillation parameters. Best-fitted parameters are $\left(\Delta m_{23}^{2}, \sin ^{2} 2 \theta_{23}\right)=$ $\left(2.2 \times 10^{-3} \mathrm{eV}^{2}, 1.0\right)$

As seen in Fig.1, allowed parameters are placed in the almost same region as the case only standard neutrino oscillation is considered[6]. Therefore, we can conclude that standard neutrino oscillation hypothesis is robust to the atmospheric neutrino data even if NSI is taken into account.

In Fig. 2, 3, and 4, we show the NSI allowed region in $\varepsilon(\mathrm{FCNC})-\varepsilon^{\prime}(\mathrm{NU})$ space and the projection of FCNC and NU plane where relative phase $\eta$ is not considered, i.e. $\eta=0$.
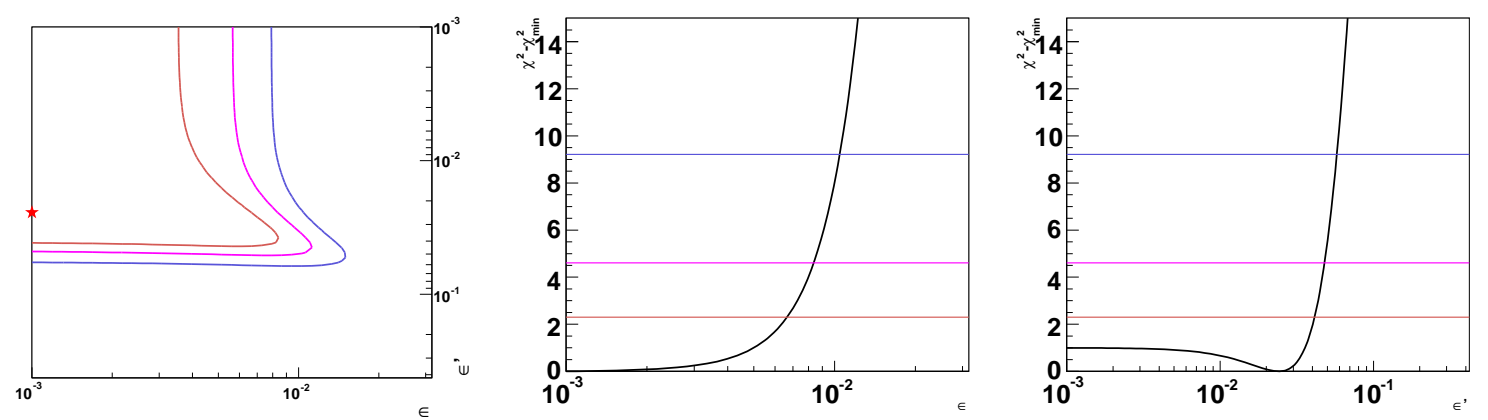

Figure 2: Allowed region of NSI Figure 3: $\Delta \chi^{2}$ as a function of Figure 4: $\Delta \chi^{2}$ as a function of NU parameters.

FCNC parameter $\varepsilon$

parameter $\varepsilon^{\prime}$

As seen in Fig. 3 and 4, NSI effects are tightly restricted less than $5.2 \times 10^{-2}$.

While, NSI effects with relative phase $\eta$ are shown in Fig. 5, 6, and 7. Contributions from NSI are still at the level of a few percents, even if relative phase is chosen arbitrary. These limits, including fixed phase, are derived from only atmospheric neutrino data without any relation between neutrino and charged lepton. Limits in both case are summarized in Table 1. 

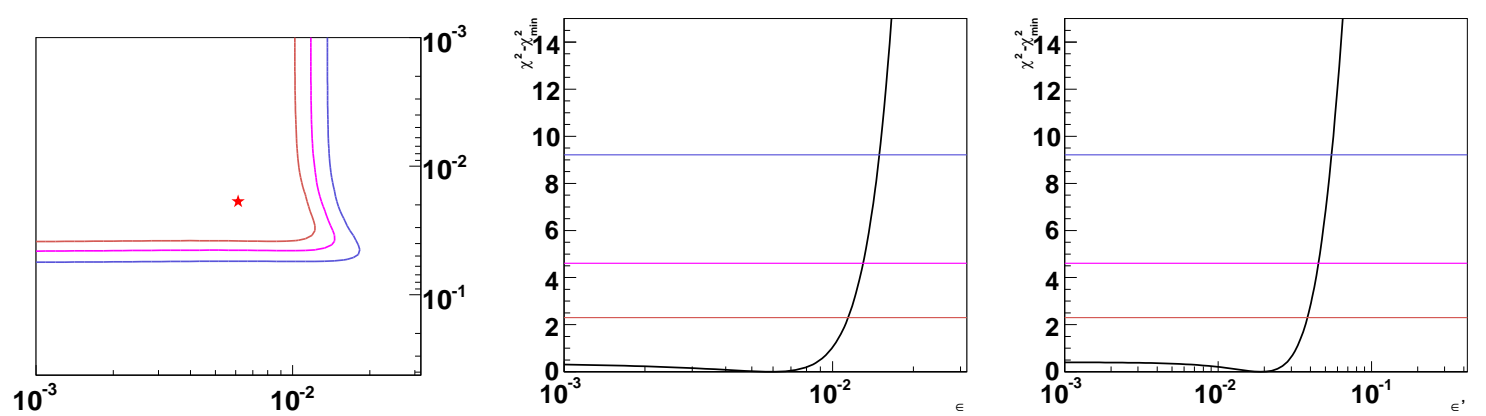

Figure 5: Allowed region of NSI Figure 6: $\Delta \chi^{2}$ as a function of Figure 7: $\Delta \chi^{2}$ as a function of NU parameters. FCNC parameter $\varepsilon$ parameter $\varepsilon^{\prime}$

\begin{tabular}{l|l|l}
\hline Relative phase $\eta$ & FCNC $\varepsilon$ & NU $\varepsilon^{\prime}$ \\
\hline \hline Fixed $(\eta=0)$ & $1.1 \times 10^{-2}$ & $5.2 \times 10^{-2}$ \\
Aribitrary & $1.5 \times 10^{-2}$ & $4.9 \times 10^{-2}$ \\
\hline
\end{tabular}

Table 1: Limits on NSI derived from SK-I and -II atmospheric neutrino data

\section{Conclusions}

2flavor oscillation analysis assuming standard oscillation+NSI hybrid model is performed with SK-I and -II FC+PC+UPMU combined data set. The best-fitted standard oscillation parameters become $\left(\Delta m_{23}^{2}, \sin ^{2} 2 \theta_{23}\right)=\left(2.2 \times 10^{-3} \mathrm{eV}^{2}, 1.0\right)$, which is almost same as pure oscillation case. The limits on NSI are $\varepsilon<1.1 \times 10^{-2}$ and $\varepsilon^{\prime}<5.2 \times 10^{-2}$ for fixed phase, $\varepsilon<1.5 \times 10^{-2}$ and $\varepsilon^{\prime}<4.9 \times 10^{-2}$ for arbitrary relative phase at $90 \%$ confidence level. The limit on FCNC are tighter than results given by the $\mathrm{NuTeV}$ experiment[7], whereas limit on NU is somewhat looser than the results from other experiment, for example the $\mathrm{NuTeV}$ experiment.

\section{Acknowledgements}

This work was supported in part by Global COE Program "the Physical Sciences Frontier", MEXT, Japan.

\section{References}

[1] P. Lipari and M. Lusignoli, Phys. Rev. D, 60, 013003 (1999).

[2] J. Schechter and J.W.F. Valle, Phys. Rev. D, 22, 2227 (1980).

[3] M.C. Gonzalez-Garcia and M. Maltoni, Phys. Rev. D, 70, 033010 (2004).

[4] M. Honda et al., Phys. Rev. D, 75, 043006 (2007).

[5] G. Mitsuka, AIP Conf. Proc. 70, 262-264 (2008).

[6] J. Raaf, Talk at the 23-th International Conference on Neutrino Physics and Astrophysics(Neutrino08), http://www2.phys.canterbury.ac.nz/ jaa53/

[7] S. Davidson et al., JHEP03, 011 (2003). 